\section{THE X-RAY SPECTROMETER.}

$\mathrm{T}$ is now well known that a homogeneous pencil of X-rays is capable of reflection by a crystal provided that the rays are directed upon the crystal at the proper angle. 1 If $\lambda$ is the wavelength of the X-rays, $d$ the spacing of the crystal planes, and $\theta$ the angle which the rays make with the planes, these quantities are connected by the relation $n \lambda=2 d \sin \theta,{ }^{2}$ where $n$ is an integer.

The object of the spectrometer is to determine the value of $\theta$ in any given case-that is to say, for a definite set of X-rays and a definite set of crystal planes. The results may be classified as follows: If we use different crystals or different faces of the same crystal, but keep the rays the same, we can compare the spacings of the various sets of planes. In this way we arrive at a knowledge of the relative positions of the atoms in the crystal-that is to say, we determine its structure.

If we use the same crystal always, but examine the angle of reflection of different homogeneous $\mathrm{X}$-rays, whether from the same or from different sources, we have the means of comparing the wave-lengths of those rays. We can, in fact, analyse X-radiation in exactly the same way as an ordinary spectrometer analyses light.

The new instrument resembles the ordinary spectrometer in its general construction. To the collimator corresponds a set of narrow slits limiting a pencil of X-rays, which is directed so as to pass through the axis of the instrument. A crystal takes the place of the diffraction grating, and is mounted on a small revolving table. The crystal face or set of planes which is acting as reflector is made to contain the direction of the axis of the instrument, and the crystal is turned round the axis until the face makes the proper angle with the incident pencil. The reflected ray then enters a cylindrical ionisation chamber filled with gas which it ionises. The chamber takes the place of the ordinary telescope, and the measurement of the ionisation current by an electroscope corresponds to observation by eye or by the photographic plate.

In the drawing of Fig. $I,{ }^{3}$ which shows the arrangement of the apparatus in plan, $Q$ is the antikathode of the X-ray bulb. The construction of the bulb is a little unusual in that the antikathode is placed perpendicularly to the kathode ray stream; the bulb can therefore be conveniently arranged so that the $\mathrm{X}$-rays leave the antikathode at a grazing angle. The finer the angle which the rays make with the antikathode, the more nearly does the source become, effectively, a "bright" line; and the narrower the line the brighter it becomes, because the "whole illumination" given out in any direction by the spot on the antikathode is independent of the direction.

I A summary of the principles on which this experimen is basef, and of the progress of its development may be found in NATURE of July 9, 1914, p. 4 CA.

2 See Proc. Camb. Phil. Soc., November rr, rgr2, or Proc. Roy. Soc. April 7 , ror 3 .

3 From a book now in the press, to be pu'llished by Messrs. G. Bell and Sons.

No. 2347 , VOL. 94]
The law followed is not that of the illumination by a surface of uniform luminosity, but rather that of the illumination due to a number of separate sources lying in one plane, each radiating uniformly in all directions. It corresponds to the case described by Rutherford, in which $\alpha$ rays are radiated from a uniform thin sheet of radio-active matter spread upon a plane surface (Phil. Mag., August, I906). The arrangement is of considerable value; the more nearly is the source a bright line parallel to the slit, the "purer" is the spectrum.

The X-ray bulb is enclosed in a wooden box heavily coated with lead. The object is to protect not merely the observer but also the sensitive apparatus. The slit through which the rays pass is only a few millimetres long and very narrow, sometimes no more than a tenth of a millimetre

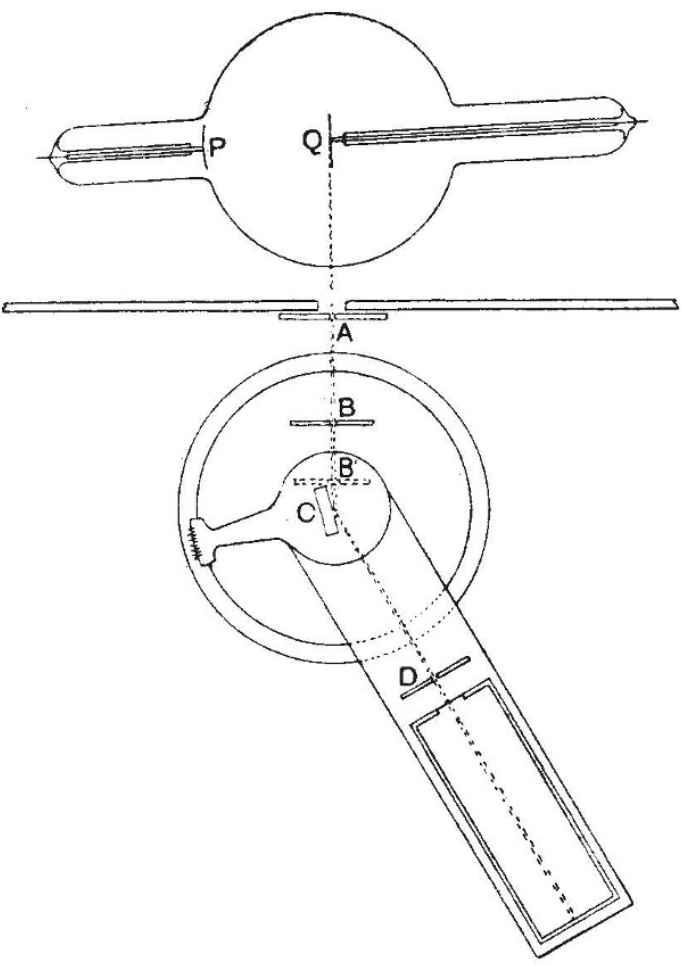

FiG. r.-X-ray spectrometer. Plan of general arrangements.

wide. Only a small fraction of the pencil that emerges is reflected in the best of circumstances, so that it is necessary to screen off all stray radiation with great care; it must be small in comparison with the radiation which is to be measured. The side of the box is shown on the left of the photograph of Fig. 2. Adjustable slits are placed at $A$ where the rays leave the box, and again at $B$, the second slit being also capable of a movement which brings it close up to the crystal, as at $B^{\prime}$. The crystal is shown at $C$, and a third slit, D, is placed just in front of the ionisation chamber.

The ionisation chamber is marked I in Fig. 2. It is a cylindrical brass chamber $15 \mathrm{~cm}$. long, and 
is filled with some heavy gas, so that the ionisation current may be as large as possible. Sulphur dioxide is convenient in many cases, but methyl bromide is much better for rays which can excite the bromine X-rays. Such rays are, for example, given off by antikathodes of silver, rhodium, or palladium, the latter two of which have been much used in the determination of crystal structure, because they give off intense homogeneous rays and also stand up well against the bombardment by the kathode'stream. The chamber I is insulated and maintained at a high potential, which drives any ionisation on to an internal electrode. The latter is connected by a fine wire which passes down inside the metal shield WW to the gold leaf electroscope (Wilson pattern) within the shield $E$. The connection with the electroscope terminal is made at a point lying on the axis of

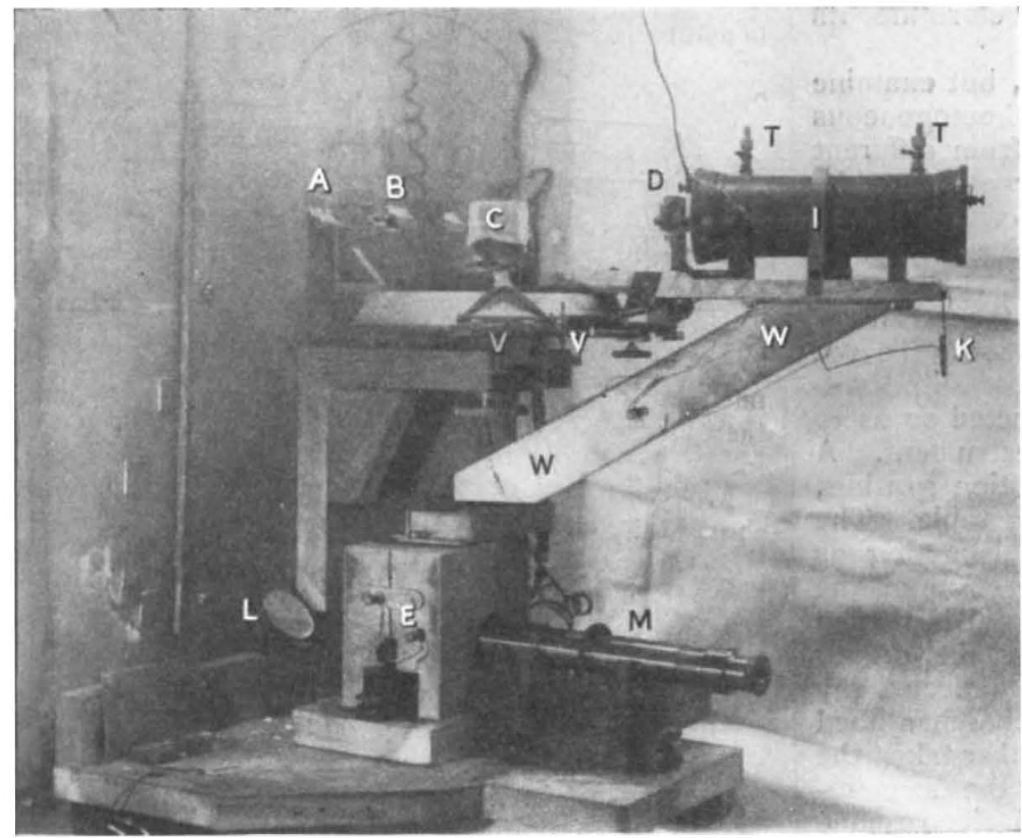

FIG, 2.-Photograph of X-ray spectrometer.

the spectroscope, so that the ionisation chamber $I$, the casing $W$, and the connecting wire may all revolve together about the spectrometer axis without straining the connection. The shielding is made solid and strong: it is necessary that the electrostatic screening should be perfect, and the electroscope must be protected from draughts which may cause changes in temperature.

The gold leaf is illuminated by reflection from a mirror L, and viewed through a microscope $M$. A strong X-ray reflection will cause the leaf to move twenty or thirty scale divisions in a seconri.

The angular positions of the crystal and the ionisation chamber are read from verniers at $\mathrm{V}$ and $\mathrm{V}^{\prime}$. Observations can easily be made to half a minute of arc, and much finer work could certainly be done, if it were required. The actual angle of reflection can be measured with an accuracy higher than can be reached in our know-
$1 \in$ dge of certain other data used in some of the calculations; for example, the actual weights of the atoms.

Crystals are often very imperfect in construction, consisting rather of a conglomerate of smaller crystals in more or less imperfect alignment. It is interesting to observe that the spectrometer may be used in ways which almost completely overcome the evil effects of the imperfections. In the case of a very perfect crystal like the diamond, the slit at $A$ is not used, but $B$ is set very fine; $D$ is wide open. The crystal is siowly turned by a tangential screw attached to the revolving table, and there is no reflection at all outside very narrow limits. The angle of maximum intensity of reflection can easily be determined to a few seconds of arc. But a crystal of rocksalt cannot be treated in this way. It is best to set $A$ and $D$ fairly fine and not to use $B$ at all. On account of certain most fortunate geometrical considerations, a homogeneous pencil of some divergence issuing from A and reflected at various points on the crystal face is brought to a line focus at $\mathrm{D}$, provided that $\mathrm{A}$ and $\mathrm{D}$ are at equal distances from the crystal (Proc. Roy. Soc., 1xxxviii., p. 433). A perfect crystal would reflect such a pencil only along a certain vertical line on the crystal face; but a poor crystal, like rocksalt, at a number of separate points on the face. Even crystals which are scarcely recognisable as such may be treated by this method.

The higher orders of spectra, that is to say, reflections at angles for which $n$ has a large value, three, four, or five, naturally give more accurate values than lower orders, though the intensity diminishes rapidly as $n$ increases. The "resolving power" increases even faster than $n$, since $d \theta i d \lambda$ is easily seen to be equal to $\tan \theta / \lambda$, which becomes very large as $\theta$ approaches the value $\pi / 2$. For example, a certain pair of lines emitted by a platinum antikathode are separated by thirty minutes of arc in the first order spectrum reflected by the cleavage face of the diamond, but in the third order spectrum they are two and a half degrees apart.

With a little practice it is quite easy to pick up the reflected X-ray. While the search is being made the slits are opened wide; as soon as the reflection is observed the slits are made narrow, and accurate measurement is then possible. It is a comparatively simple matter to find the angles of reflection of rays of given quality in the various faces or sets of planes of a crystal. The greater difficulty arises in the geometrical interpretation of the results.

NO. 2347, VOL. 94] 